\title{
A theoretical investigation of the stability of crystalline silicon dicarbide
}

\author{
R. C. Andrew, ${ }^{1, *}$ M. Braun,,${ }^{1, \dagger}$ and N. Chetty ${ }^{1,2,+}$ \\ ${ }^{1}$ Physics Department, University of Pretoria, Pretoria 0002, South Africa \\ ${ }^{2}$ National Institute for Theoretical Physics, Johannesburg 2000, South Africa
}

(Dated: March 20, 2012)

\begin{abstract}
While $\mathrm{Si}, \mathrm{C}$ and $\mathrm{SiC}$ are very well known materials that have been extensively studied in their multitude of structures and allotropes, there is, surprisingly, a dearth of reliable information for off-50:50 compounds involving Si and C. Do such compounds exist and, if so, what are the plausible structures for these compounds? Using first principles total energy methods, we generally explore these questions before investigating in some detail the structures involving silicon dicarbide. Of the structures considered, our results show that the tetragonal glitter phase is lowest in energy. Of the two higher energy cubic structures that we considered, we note that there is a continuous transition from the high energy fluorite structure to the lower energy pyrite structure. We predict the transition pressure from glitter to pyrite to be $24.7 \mathrm{GPa}$. We give detailed structural and electronic properties for these systems. Since diamond and $\mathrm{SiC}$ are known for their hardness properties, we compute the elastic properties of $\mathrm{SiC}_{2}$, and we make an assessment of its hardness properties. We propose the glitter structure to be a plausible phase for $\mathrm{SiC}_{2}$.

PACS numbers: 61.66.Dk, 62.20.de, 71.20.Nr
\end{abstract}

\section{INTRODUCTION}

Silicon and carbon have both been at the very forefront in the technological advancements of the last century, and applications are set to continue to grow at a rapid rate into the future. Si has an established history with semiconductor applications, while $\mathrm{C}$ has been the subject of very extensive research over a great many decades because of its propensity to form different allotropes such as graphite, buckeyballs, nanotubes, graphene and diamond with extreme properties. Recently, Umemoto et al. [1] predicted the metastability at ambient conditions of a new form of $\mathrm{C}$ based on density functional computations. Diamond is often used as the defining standard for hardness and has found useful applications, for example, as an abrasive in the cutting tool industry.

It is fascinating that despite the similarity of these two systems, for example in terms of the structure (diamond), valency and electronic structure, there is a great dearth in the availability of reliable data for alloys involving these two elements. Of course, the 50:50 alloy of silicon carbide in its cubic and various hexagonal stacking polytypes are very well known and have been well studied using ab initio techniques [2-6]. Applications involving $\mathrm{SiC}$ also center on hardness and thermal properties, for example in applications involving powder technology such as high-tech ceramics [7], high-power devices [8] and nuclear fuel casings [9].

However, it remains a great mystery why alloys of the form $\mathrm{Si}_{1-x} \mathrm{C}_{x}$ with $0.1 \lesssim x \lesssim 0.4$ or $0.6 \lesssim x \lesssim 0.9$, which we refer to as off-50:50 alloys, do not seem to form with ease. Empirical evidence suggests that there is a low solubility of $\mathrm{C}$ in $\mathrm{Si}[10]$ and low concentrations of metastable $\mathrm{C}$ defects in $\mathrm{Si}$ have been created experimentally using molecular beam epi-

\footnotetext{
*Richard.Andrew@up.ac.za

${ }^{\dagger}$ Max.Braun@up.ac.za

‡Nithaya.Chetty@up.ac.za
}

taxy $[11,12]$ and chemical vapor deposition [13] and studied theoretically [14-16], but off-50:50 alloys do not seem to readily exist. An extensive search of the literature shows a dearth of ordered, disordered, random, as well as amorphous and thin film structures [17] for these alloy concentrations.

Clearly, the reasons for the apparent immiscibility must center more on the differences between $\mathrm{Si}$ and $\mathrm{C}$ rather than the similarities between these two elements. What is known is that $\mathrm{C}$ has a preponderance for forming $\mathrm{sp}^{2}$ and $\mathrm{sp}^{3}$ bonds, whereas Si prefers $\mathrm{sp}^{3}$ bonds under ambient conditions. All the 50:50 alloys of $\mathrm{SiC}$ under ambient conditions exhibit tetrahedral coordination which suggests that in an alloyed configuration under ambient conditions, $\mathrm{sp}^{3}$ bonding may also be preferred.

Is it possible to create off-50:50 alloys where the atoms maintain their tetrahedral coordination? Given the sizeable difference in the bond lengths of these two elements in the diamond structure (the bond length of $\mathrm{C}$ is about $\frac{2}{3}$ that for $\mathrm{Si}$ ), it is clear that this will be difficult. The 50:50 alloys are structured only on homogeneous $\mathrm{Si}-\mathrm{C}$ bonds, which explains in part their stability. The presence of $\mathrm{Si}-\mathrm{Si}, \mathrm{C}-\mathrm{C}$ and $\mathrm{Si}-\mathrm{C}$ bonds all in any single system will result in strains which could, in principle, only be relieved in complex geometries. Alternatively, amorphous or disordered structures could result.

Off-50:50 systems for which the Si atom attains its preferred $\mathrm{sp}^{3}$ form while the $\mathrm{C}$ atom is free to form $\mathrm{sp}^{3}$ and/or $\mathrm{sp}^{2}$ bonds perhaps in layered geometries are yet to be found. Are ordered alloys of these forms possible? Are the amorphous forms more realistic? Could such thin alloy films be synthesized at surfaces or interfaces?

It turns out that under conditions of high pressure, $\mathrm{SiC}$ transforms to the rock-salt structure [18], which is sixfold coordinated and comprises homogeneous Si-C bonds. Pressure would be a useful tool to search for off-50:50 alloys involving Si and C. Grumbach and Martin [19], in their theoretical work on high-pressure/high-temperature phases of $\mathrm{C}$, discovered a dense liquid whose melting temperature decreased with pressure, which is a behavior that is very similar to that of $\mathrm{Si}$ and 
$\mathrm{Ge}$ at lower pressures. This liquid resembles the simple cubic structure with sixfold coordination, a structure similar to that of $\mathrm{Si}$ and $\mathrm{Ge}$ under pressure. This gives us some insights into the possibilities for alloy formation of these systems. It could be inferred, for instance, that simple high coordination ordered structures might form under conditions of high pressure. It is also clear that temperature is a useful tool to explore such systems.

There are opportunities here for both experimentalists and theorists to address. In this paper, we employ density functional theory within the generalized gradient approximation to consider some of the questions that we have posed. These methods have been shown to be very accurate in describing many material systems and especially those involving $\mathrm{Si}$ and C $[20,21]$ and including, more recently, the Van der Waals interaction[22] that is prevalent in graphite.

In section II, we begin with a general exploration of the question of the possible existence of off-50:50 alloys involving $\mathrm{Si}$ and $\mathrm{C}$, and we then focus specifically on our proposed structures of $\mathrm{SiC}_{2}$. In section III we present details of our computational methods. In section IV we present our results. We give detailed arguments why the glitter phase is the most plausible structure. We draw our conclusions with suggestions for possible future work in section $\mathrm{V}$.

\section{OFF-50:50 ALLOYS INVOLVING Si AND C}

\section{A. Preamble}

The tools that we have available to investigate the possible structures of off-50:50 are wide ranging. Even before beginning our computations, as a guiding rule, we consider systems for which $d_{S i-S i} \geq d_{S i-C} \geq d_{C-C}$, where $d$ is the bond length. Our initial arguments are based on geometry and symmetry. For example, the perovskite-derived structures were considered in our initial investigations. $\mathrm{SiC}_{4}, \mathrm{Si}_{2} \mathrm{C}_{3}, \mathrm{Si}_{3} \mathrm{C}_{2}$ and $\mathrm{Si}_{4} \mathrm{C}$ were studied. Applying the above rule for the ordering of the bond lengths, we were very quickly able to discount a number of the structures. Only $\mathrm{Si}_{4} \mathrm{C}$ with $\mathrm{Si}$ atoms on the corners and face centers and $\mathrm{C}$ in the body center emerged as a possibility, which was then subjected to further computational scrutiny and subsequently rejected. This was our general approach in considering a variety of different structures. In our circumscribed investigation, we were keen to explore open, covalently bonded structures, and C-layered structures. As already indicated in the introduction, high coordination structures could be accessible under conditions of pressure. Dense structures derived, for example, from the bi-metallics $\mathrm{Cu}_{3} \mathrm{Au}$, $\mathrm{Cr}_{3} \mathrm{Si}, \mathrm{CsCl}$ and $\mathrm{CuAu}$ would be some systems to consider, but an extensive investigation of such systems was beyond the scope of our current investigation.

Using first principles methods, we calculated heats of formation and equilibrium structures for systems for a range of structures and stoichiometries. For systems within the same stoichiometry, we computed enthalpy versus pressure curves and transition pressures. In some cases we discounted structures based on high negative transition pressures. We com- puted elastic constants and phonon frequencies, negative results of which indicated system instabilities. This further reduced our set of plausible structures. We compared our results with $\mathrm{Si}, \mathrm{C}$ and $\mathrm{SiC}$ whenever possible. We calculated electronic band structures to investigate the electronic properties of these systems.

Presenting results for all systems that we tested - many of which were quickly rejected based on the above arguments - is not considered in this paper. In the rest of this paper, we focus our attention on silicon dicarbide, which we concluded is a plausible material. Our results showed up $\mathrm{Si}_{2} \mathrm{C}$ as not being favorable.

\section{B. Proposed structures for $\mathrm{SiC}_{2}$}

Recently, graphene, nanotube and nanoribbon structures for $\mathrm{SiC}_{2}$ have been proposed and studied [23]. We propose to study two crystalline structures: a previously suggested tetragonal structure [24] derived from a theoretical structure for C called glitter [25], and the cubic pyrite structure based on $\mathrm{FeS}_{2}$.

The pyrite structure shown in Fig. 1 has space group $P a \overline{3}$. It is composed of $\mathrm{Si}$ atoms at FCC sites with $\mathrm{C}$ atoms placed at lattice position $(x, x, x)$ and at all symmetry-equivalent locations to $(x, x, x)$ in units of the lattice constant. The pyrite structure is identical to the fluorite structure, shown in Fig. 2, when $x=0.25$. Each $\mathrm{C}$ atom has 4 -fold coordination while each Si atom has 6-fold coordination.

In the search for hypothetical structures for $\mathrm{B}_{2} \mathrm{O}$, Ref. 26 found two structures similar to glitter where the 4-fold coordinated $\mathrm{C}$ atoms of glitter are replaced by $\mathrm{B}$ atoms and half the 3 -fold coordinated $\mathrm{C}$ atoms are replaced by $\mathrm{O}$ giving space groups of $P 4_{2} m c$ and $P-4 m 2$. For our tetragonal structure, all the 4-fold coordinated $\mathrm{C}$ atoms are replaced with $\mathrm{Si}$ atoms giving it the space group $P 4_{2} / m m c$. This structure (henceforth referred to as the glitter structure) is shown in Fig. 3 and has atomic positions as given in Table. I. For this structure, each $\mathrm{C}$ atom has 3-fold coordination.

There are both Si-C and C-C bonds in these systems.

\section{THEORY AND METHODOLOGY}

All calculations were done using the VASP [27] software code which implements density functional theory [28] (DFT) using the projector augmented-wave (PAW) method [29]. The PBEsol exchange-correlation functional [30] of the generalized gradient approximation (GGA) was used to accurately describe the bulk properties [31] of the $\mathrm{SiC}_{2}$ systems using PAW pseudopotentials [32]. A kinetic-energy cut-off of $800 \mathrm{eV}$ was used to obtain highly converged total energies. Monkhorst-Pack [33] grids were used to sample the Brillouin zone and in order to achieve a consistent sampling density, a grid size of $11 \times 11 \times 11$ was used for the pyrite structure and $17 \times 17 \times 9$ for the glitter structure. All single-point total energy, band structure and electronic density of states 
(DOS) calculations were done using the tetrahedron integration method with Blöchl corrections [34]. For geometrical optimizations, a Methfessel-Paxton smearing [35] with a width of $0.2 \mathrm{eV}$ was used to integrate the bands at the fermi level.

Elastic constants were obtained using the method of leastsquares fit [36] as implemented in the MedeA-MT module while using the tetrahedron method of k-point integration. The eigenvalues of the stiffness matrix were calculated to determine the mechanical stability of the structure. The MT module further used the fact that the bulk and shear elastic moduli of polycrystalline material are bounded [37] above by the Voigt values

$$
\begin{gathered}
B_{V}=\frac{1}{9}\left(C_{11}+C_{22}+C_{33}\right)+\frac{2}{9}\left(C_{12}+C_{13}+C_{23}\right) \\
G_{V}=\frac{1}{15}\left(C_{11}+C_{22}+C_{33}\right)-\frac{1}{15}\left(C_{12}+C_{13}+C_{23}\right)+\frac{1}{5}\left(C_{44}+C_{55}+C_{66}\right)
\end{gathered}
$$

and below by the Reuss values

$$
\begin{gathered}
1 / B_{R}=\left(S_{11}+S_{22}+S_{33}\right)+2\left(S_{12}+S_{13}+S_{23}\right) \\
15 / G_{R}=4\left(S_{11}+S_{22}+S_{33}\right)-4\left(S_{12}+S_{13}+S_{23}\right)+3\left(S_{44}+S_{55}+S_{66}\right)
\end{gathered}
$$

where $S_{i j}$ denotes the elements of the compliance matrix. It used the geometric mean of the two limits (the Hill value) as a reasonable approximation to describe the polycrystalline material. These values are then used to calculate the longitudinal, shear and mean sound velocities as well as the Debye temperature using the method described in Ref. 38.

Each structure was fully relaxed at various pressures to obtain third-order Birch equation of state [39] fits as well as enthalpy vs pressure curves for each structure phase.

Phonon calculations were done using the general direct approach of lattice dynamics as implemented in the MedeAPHONON package [40].

\section{RESULTS AND DISCUSSION}

The fluorite structure is a special case of the pyrite structure with $x=0.25$. In Fig. 4 we plot the total energy of the pyrite structure as a function of the internal parameter $x$. For each value of $x$, we compute the lowest energy corresponding to the equilibrium cell volume, making a continuous transition over all possible pyrite structures. We find a local minimum in the energy for the fluorite structure and an equilibrium structure corresponding to a global minimum at $x=0.417$ with an energy difference between these two structures of $3.28 \mathrm{eV}$ per formula unit.

However, an analysis of the fluorite structure shows it to be dynamically unstable: the phonon dispersion spectra in Fig. 5 indicates the presence of soft modes. The pyrite structure at the global minimum (henceforth referred to as the pyrite structure), on the other hand, is stable at zero pressure as indicated by positive phonon frequencies shown in Fig. 6. The pyrite structure is therefore a potential candidate system for $\mathrm{SiC}_{2}$, which we explore further.

The Birch fit parameters for the pyrite structure along with the least-squares fitted elastic constants are shown in Table. II. The relaxed pyrite structure has a lattice constant of $a=4.754 \AA$ giving a density of $3.221 \mathrm{~g} \mathrm{~cm}^{-3}$ with the angles $\mathrm{Si}-\hat{\mathrm{C}}-\mathrm{Si}=109.34^{\circ}$ and $\mathrm{C}-\hat{\mathrm{C}}-\mathrm{Si}=109.60^{\circ}$ giving a slightly distorted tetrahedral nature to the bonding around each $\mathrm{C}$ atom (refer to Fig. 7). Through each $\mathrm{Si}$ atom, the opposing $\mathrm{Si}-\mathrm{C}$ bonds form three straight $\mathrm{C}$-Si-C segments which are not fully orthogonal to each other but either form an angle of $87.91^{\circ}$ or the complimentary angle of $92.09^{\circ}$, giving a distorted octahedral character to the symmetry. The bond distances are $d_{C-C}=1.363 \AA$ (shorter than that in diamond where $d_{C-C}=1.545 \AA$ ), while $d_{S i-C}=2.060 \AA$ (larger than that in cubic $\mathrm{SiC}$ where $d_{S i-C}=1.888 \AA$.) The single-crystal bulk modulus using the calculated elastic constants is $194.0 \mathrm{GPa}$ in reasonable agreement with the Birch value of $192.3 \mathrm{GPa}$. The eigenvalues of the stiffness matrix are all positive indicating mechanical stability.

For the glitter structure, Ref. 24 obtained cell length parameters of $a=3.07 \AA$ and $c / a=2.287$ and calculated a bulk modulus of $230 \mathrm{GPa}$ using a semi-empirical formula [41] with assumed bond lengths of $d_{S i-C}=1.88 \AA$ and $d_{C-C}=1.34 \AA$ based on a DFT geometric optimization of a 1,4-disilaquinoid molecule. Our Birch fit gives values of $a=3.156 \AA$ and $c / a=2.179$ giving a density of $2.527 \mathrm{~g} \mathrm{~cm}^{-3}$ with a lower single-crystal bulk modulus of $163.8 \mathrm{GPa}$ which is in agreement with the value of $163.7 \mathrm{GPa}$ obtained from our calculated elastic constants. We found similar bond lengths of $d_{C-C}=1.364 \AA$ (shorter than that in diamond) and $d_{S i-C}=1.888 \AA$ (identical to that in cubic SiC). The distorted tetrahedral bonding around each $\mathrm{Si}$ atom, as shown in Fig. 7, has two angles of $\mathrm{C}-\hat{\mathrm{Si}}-\mathrm{C}=113.40^{\circ}$ and another four of $\mathrm{C}-\mathrm{Si}-\mathrm{C}=107.54^{\circ}$. This creates distorted trigonal bonding around each $\mathrm{C}$ atom with $\mathrm{Si}-\hat{\mathrm{C}}-\mathrm{Si}=113.40^{\circ}$ and $\mathrm{Si}-\hat{\mathrm{C}}-\mathrm{C}=123.30^{\circ}$. The eigenvalues of the stiffness matrix are all positive and the phonon dispersion plot in Fig. 8 shows no 
soft modes, indicating mechanical stability at zero pressure.

The band structures and DOS for the glitter structure, as shown in Fig. 9, and for the pyrite structure, as shown in Fig. 10, both indicate that the two forms of $\mathrm{SiC}_{2}$ are metallic. The density of electronic states at the Fermi level is greater for the pyrite structure compared with the glitter structure which has a depression in the density of states at the Fermi level. This lends credence to the fact that the glitter structure is energetically more favorable compared with the pyrite structure, a result borne out by the calculation of the energy of formation, which we discuss a little later. The pyrite structure has an energy gap above the Fermi energy; the high lying states above the gap have the potential to overlap with the conduction band states under pressure. This suggests that the pyrite structure is not a simple metal. The metallic nature of the electronic states gives rise to ductile behavior and consequently lower elastic moduli compared with the 50:50 alloys, this despite the higher $\mathrm{C}$ content and the shorter $\mathrm{C}-\mathrm{C}$ bond lengths in both structures for $\mathrm{SiC}_{2}$.

By relaxing the two structures under pressure, the enthalpy versus pressure plot in Figure. 11 was obtained. It is clear that the glitter structure is the stable phase at low pressure until a transition to the pyrite phase at 24.7 GPa. The energy difference between these phases at zero pressure is $1.02 \mathrm{eV}$ per formula unit. The heats of formation were calculated by comparing the energies of glitter and pyrite with the bulk elemental phases for Si and C. Since GGA does not adequately describe the inter-layer Van der Waals interaction, the energy for $\mathrm{C}$ in graphite was calculated by fixing the lattice parameters to the experimental values. Our results for the heats of formation are $\Delta \mathrm{H}_{\text {Glitter }}=124.6 \mathrm{~kJ} \mathrm{~mol}^{-1}$ and $\Delta \mathrm{H}_{\text {Pyrite }}=223.3 \mathrm{~kJ} \mathrm{~mol}^{-1}$. The positive heats of formation indicate that these phases do not form spontaneously under ambient conditions. We suggest that C-rich conditions of growth under pressure and temperature are needed to explore the synthesis of this new material. It is contemplated that pyrite forms before glitter under conditions of pressure and temperature, and that the glitter phase is subsequently stabilized by the reduction of temperature before the reduction of pressure.

The calculated Hill elastic moduli, Poisson's ratio and sound velocities for both structures are given in Table. III. The table shows increasing values for the bulk modulus as one moves down the columns with tetrahedrally-bonded $\mathrm{SiC}$ having the highest value, pyrite a value of $86 \%$ of $\mathrm{SiC}$ and glitter $76 \%$. The pyrite structure has a higher value than that of glitter due to the fact that it contains $\mathrm{C}$ atoms in the center of near-perfect tetrahedra and $\mathrm{Si}$ atoms in the center of near-perfect octahedra. This gives the structure a geometrical configuration that is more resistant to isotropic compressive strain compared to glitter with its very distorted tetrahedral and trigonal bonding.

The shear modulus for $\mathrm{SiC}$ is the highest, with pyrite having a value of only $50 \%$ that of $\mathrm{SiC}$, and glitter $28 \%$. The Young's modulus for $\mathrm{SiC}$ is also the highest with pyrite having a value $55 \%$ that of $\mathrm{SiC}$, and glitter $32 \%$. The low shear modulus for the glitter structure is due to its greater anisotropy. The low value for its Young's modulus is due to bending of the trigonal bonds around each $\mathrm{C}$ atom. Although the Poisson's ratios for both $\mathrm{SiC}_{2}$ structures are similar, they are sig- nificantly greater than that for $\mathrm{SiC}$ suggesting $\mathrm{SiC}_{2}$ to have a higher ductility [42]. This is consistent with the metallic nature of both $\mathrm{SiC}_{2}$ structures. The large value for glitter is due to a decrease in the $\mathrm{Si}-\hat{\mathrm{C}}-\mathrm{Si}$ angles causing a significant reduction in the cross-sectional area of the unit cell when under strain in the [001] direction. Due to the trends in the bulk and shear moduli, the mean acoustic velocities also increase as one goes down the columns with the value for pyrite having a value $72 \%$ that of $\mathrm{SiC}$, and glitter $61 \%$. Higher velocities are indicative of an increase in stiffness.

\section{CONCLUSIONS}

Our work is motivated by the search for off-50:50 alloys involving $\mathrm{Si}$ and $\mathrm{C}$. We considered a number of different structures and stoichiometries involving these elements. We used arguments based on the ordering of bond lengths, energetics, transition pressures, elastic constants and phonon frequencies to reduce the set of plausible structures. We honed in on $\mathrm{SiC}_{2}$ as showing the greatest promise as a new material that could be synthesized.

We found the fluorite structure for $\mathrm{SiC}_{2}$ to be dynamically unstable. The equilibrium structure for the pyrite structure corresponds to an internal parameter of $x=0.417$. The transition pressure from the glitter to the pyrite phase was calculated to be $24.7 \mathrm{GPa}$. We found the pyrite phase to have a higher enthalpy of formation compared with the glitter phase.

Both the glitter and pyrite structures are found to be dynamically stable at zero pressure as indicated by positive elastic moduli, phonon frequencies and eigenvalues of the stiffness matrix. Both systems exhibit metallic character. We predict the pyrite phase to not be a simple metal because of the existence of an energy band gap above the Fermi level. The metallic nature of these materials manifests itself in elastic moduli that are considerably lower that those for the 50:50 alloys despite the higher $\mathrm{C}$ content and shorter $\mathrm{C}-\mathrm{C}$ bond lengths.

We propose that the pyrite phase can be synthesized under C-rich conditions under pressure and temperature, and the glitter phase may be stabilized by reducing temperature before pressure.

We suggest that a detailed understanding of these phases is essential to understand the potential for the multi-phase softening of the technologically important material of SiC.

Future work in this area centers on the investigation of other stoichiometries involving high-coordinated systems that are accessible under pressure.

\section{ACKNOWLEDGMENTS}

Richard Andrew expresses gratitude to the National Institute for Theoretical physics for financial support and Dr Maria Entrialgo Castaño of Materials Design for help with the MT calculations. The authors would like to thank Prof Jannie Pretorius for his assistance with the use of the VASP electronic structure codes. 
[1] K. Umemoto, R. M. Wentzcovitch, S. Saito, T. Miyake, BodyCentered Tetragonal $\mathrm{C}_{4}$ : A Viable $s p^{3}$ Carbon Allotrope, Physical Review Letters 104 (12) (2010) 125504.

[2] C. H. Park, B.-H. Cheong, K.-H. Lee, K. J. Chang, Structural and electronic properties of cubic, $2 \mathrm{~h}, 4 \mathrm{~h}$ and $6 \mathrm{~h}$ sic, Physical Review B 49 (7) (1994) 4485.

[3] P. Käckell, B. Wenzien, F. Bechstedt, Electronic properties of cubic and hexagonal $\mathrm{SiC}$ polytypes from ab initio calculations, Physical Review B 50 (15) (1994) 10761.

[4] K. J. Chang, M. L. Cohen, Ab initio pseudopotential study of structural and high-pressure properties of SiC, Physical Review B 35 (15) (1987) 8196.

[5] K. Karch, F. Bechstedt, P. Pavone, D. Strauch, Pressuredependent properties of SiC polytypes, Physical Review B 53 (20) (1996) 13400.

[6] K. Karch, P. Pavone, W. Windl, O. Schütt, D. Strauch, Ab initio calculation of structural and lattice-dynamical properties of silicon carbide, Physical Review B 50 (23) (1994) 1705417063.

[7] S. C. Zhang, G. E. Hilmas, W. G. Fahrenholtz, Mechanical properties of sintered ZrB_2 - SiC ceramics, Journal of the European Ceramic Society 31 (2011) 893.

[8] P. Friedrichs, SiC power devices for industrial applications, International Power Electronics Conference (IPEC) - ECCE Asia (2010) 3241.

[9] L. L. Snead, T. Nozawa, Y. Katoh, T.-S. Byun, S. Kondo, D. A. Petti, Handbook of SiC properties for fuel performance modeling, Journal of Nuclear Materials 371 (1-3) (2007) 329.

[10] D. H. Hofmann, M. H. Müller, Prospects of the use of liquid phase techniques for the growth of bulk silicon carbide crystals, Materials Science and Engineering: B 61-62 (1999) 29.

[11] M. W. Dashiell, L. V. Kulik, D. Hits, J. Kolodzey, G. Watson, Carbon incorporation in $\mathrm{Si}_{-} 1-y \mathrm{C}_{-} y$ alloys grown by molecular beam epitaxy using a single silicon-graphite source, Applied Physics Letters 72 (7) (1998) 833.

[12] K. Eberl, K. Brunner, W. Winter, Pseudomorphic Si_1 - yC_y and $\mathrm{Si}_{-} 1-x-y \mathrm{G} e_{-} x \mathrm{C}_{-} y$ alloy layers on $\mathrm{Si}$, Thin Solid Films 294 (1997) 98.

[13] K. H. Chung, J. C. Sturm, E. Sanchez, K. K. Singh, S. Kuppurao, The high growth rate of epitaxial silicon-carbon alloys by using chemical vapour deposition and neopentasilane, Semiconductor Science and Technology 22 (1) (2007) S158.

[14] A. A. Demkov, O. F. Sankey, Theoretical investigation of random Si-C alloys, Physical Review B 48 (4) (1993) 2207.

[15] A. Yakoubi, L. Beldi, B. Bouhafs, M. Ferhat, Structural, electronic and energetic properties of silicon carbon alloys, Physica B 388 (2007) 167.

[16] H. Rücker, M. Methfessel, E. Bugiel, H. J. Osten, StrainStabilized Highly Concentrated Pseudomorphic Si $1-x \mathrm{C}_{-} x$ Layers in Si, Physical Review Letters 72 (22) (1994) 3578.

[17] T. Maruyama, The network structure of amorphous siliconcarbon alloy, Journal of Non-Crystalline Solids 319 (3) (2003) 219-224.

[18] M. Yoshida, A. Onodera, M. Ueno, K. Takemura, O. Shimomura, Pressure-induced phase transition in SiC, Physical Review B 48 (14) (1993) 10587.

[19] M. P. Grumbach, R. M. Martin, Phase diagram of carbon at high pressures and temperatures, Physical Review B 54 (22) (1996) 15730.

[20] M. T. Yin, M. L. Cohen, Theory of static structural properties, crystal stability, and phase transformations: Applications to $\mathrm{Si}$ and Ge, Physical Review B 26 (10) (1982) 5668.
[21] M. T. Yin, Si-III (BC-8) crystal phase of Si and C: Structural properties, phase stabilities, and phase transitions, Physical Review B 30 (4) (1984) 1773.

[22] G. Román-Pérez, J. M. Soler, Efficient Implementation of a van der Waals Density Functional: Application to Double-Wall Carbon Nanotubes, Physical Review Letters 103 (2009) 096102.

[23] Y. Li, F. Li, Z. Zhou, Z. Chen, SiC_\{2\} Silagraphene and Its One-Dimensional Derivatives : Where Planar Tetracoordinate Silicon Happens, Journal of the American Chemical Society 133 (2011) 900.

[24] M. J. Bucknum, A. Ienco, E. A. Castro, Electronic structure and bulk modulus of silicon dicarbide: a glitter phase, Journal of Molecular Structure: THEOCHEM 716 (2005) 73.

[25] M. J. Bucknum, R. Hoffmann, A Hypothetical Dense 3,4Connected Carbon Net and Related B_2C and CN_2 Nets Built from 1,4-Cyclohexadienoid Units, J. Am. Chem. Soc. 116 (1994) 11456.

[26] Q. Li, W. Chen, Y. Xia, Y. Liu, H. Wang, H. Wang, Y. Ma, Superhard phases of B $2 \mathrm{O}$ : An isoelectronic compound of diamond, Diamond \& Related Materials 20 (4) (2011) 501.

[27] G. Kresse, J. Furthmüller, Efficient iterative schemes for ab initio total-energy calculations using a plane-wave basis set, Physical Review B 54 (16) (1996) 11169.

[28] P. Hohenberg, W. Kohn, Inhomogeneous Electron Gas, Physical Review 136 (3B) (1964) 864.

[29] P. E. Blöchl, Projector augmented-wave method, Physical Review B 50 (24) (1994) 17953.

[30] J. P. Perdew, A. Ruzsinszky, G. I. Csonka, O. A. Vydrov, G. E. Scuseria, L. A. Constantin, X. Zhou, K. Burke, Restoring the Density-Gradient Expansion for Exchange in Solids and Surfaces, Physical Review Letters 100 (2008) 136406.

[31] G. I. Csonka, J. P. Perdew, A. Ruzsinszky, P. H. T. Philipsen, S. Lebègue, J. Paier, O. Vydrov, J. Ángyán, Assessing the performance of recent density functionals for bulk solids, Physical Review B 79 (15) (2009) 155107.

[32] G. Kresse, D. Joubert, From ultrasoft pseudopotentials to the projector augmented-wave method, Physical Review B 59 (3) (1999) 1758-1775.

[33] H. J. Monkhorst, J. D. Pack, Special points for Brillouin-zone integration, Physical Review B 13 (12) (1976) 5188.

[34] P. E. Blöchl, O. Jepsen, O. K. Andersen, Improved tetrahedron method for Brillouin-zine integrations, Physical Review B 49 (23) (1994) 16223.

[35] M. Methfessel, A. T. Paxton, High-precision sampling for Brillouin-zone integration in metals, Physical Review B 40 (6) (1989) 3616.

[36] Y. Le Page, P. Saxe, Symmetry-general least-squares extraction of elastic data for strained materials from ab initio calculations of stress, Physical Review B 65 (2002) 104104.

[37] R. Hill, The elastic behaviour of a crystalline aggregate, Proceedings of the Pysical Society, Section A 65 (5) (1952) 349.

[38] O. L. Anderson, A simplified method for calculating the Debye temperature from elastic constants, J Phys Chem Solids 24 (1963) 909.

[39] F. Birch, Finite Elastic Strain of Cubic Crystals, Physical Review 71 (11) (1947) 809.

[40] K. Parlinski, PHONON Software, K. Parlinski, Z. Q. Li, Y. Kawazoe, First-Principles Determination of the Soft Mode in Cubic ZrO2, Physical Review Letters 78 (21) (1997) 4063.

[41] M. L. Cohen, Predicting useful materials, Science 261 (1993) 307-308. 
[42] S. F. Pugh, Relations between the Elastic Moduli and the Plastic Properties of Polycrystalline Pure Metals, Philosophy magazine 45 (367) (1954) 823.
[43] R. D. Carnahan, J. Am. Ceramic Soc. 51 (1968) 223. 
TABLE I. Atomic positions in the tetragonal glitter cell as ratios of the cell length parameters $a$ and $c$.

\begin{tabular}{rccc}
\hline \hline atom & $\mathrm{x} / \mathrm{a}$ & $\mathrm{y} / \mathrm{a}$ & $\mathrm{z} / \mathrm{c}$ \\
\hline $\mathrm{Si}$ & 0 & 0 & $1 / 4$ \\
$\mathrm{Si}$ & 0 & 0 & $3 / 4$ \\
$\mathrm{C}$ & 0 & $1 / 2$ & $6 / 10$ \\
$\mathrm{C}$ & 0 & $1 / 2$ & $4 / 10$ \\
$\mathrm{C}$ & $1 / 2$ & 0 & $9 / 10$ \\
$\mathrm{C}$ & $1 / 2$ & 0 & $1 / 10$ \\
\hline \hline
\end{tabular}

TABLE II. Lattice parameters $a$ and $c / a$, density $\rho$, bulk modulus $B$, pressure derivative $B^{\prime}$ from Birch fits (lattice constants in $\AA$, density in $\mathrm{g} \mathrm{cm}^{-3}$, bulk modulus in GPa) and elastic constants $C_{i j}$ calculated from a least-squares fit (values in GPa with stated error bars indicating the numerical uncertainty of the fit).

\begin{tabular}{|c|c|c|c|c|c|c|c|c|c|c|c|c|}
\hline & $a$ & $c / a$ & $\rho$ & B & $B^{\prime}$ & $C_{11}$ & $C_{12}$ & $C_{13}$ & $C_{16}$ & $C_{33}$ & $C_{44}$ & $C_{66}$ \\
\hline glitter & 3.156 & 2.179 & 2.527 & 163.8 & 3.28 & $333.49 \pm 1.20$ & $15.40 \pm 1.20$ & $101.51 \pm 0.85$ & $0.31 \pm 1.00$ & $521.85 \pm 1.20$ & $33.16 \pm 1.70$ & $8.39 \pm 1.70$ \\
\hline pyrite & 4.754 & & 3.221 & 192.3 & 4.64 & $415.76 \pm 1.30$ & $83.05 \pm 1.30$ & & & & $65.89 \pm 1.84$ & \\
\hline
\end{tabular}

TABLE III. Calculated elastic moduli based on elastic constants for both structures showing upper bound Voigt, lower bound Reuss and geometric mean Hill values in GPa along with Poisson's ratio $\sigma$ and the shear, longitudinal and mean sound velocities $v$ (in $m s^{-1}$ ). Debye temperature in Kelvin.

\begin{tabular}{|c|c|c|c|c|c|c|c|c|c|c|c|c|c|c|}
\hline & $B_{V}$ & $B_{R}$ & $B_{H}$ & $G_{V}$ & $G_{R}$ & $G_{H}$ & $Y_{V}$ & $Y_{R}$ & $Y_{H}$ & $\sigma$ & $v_{s}$ & $v_{l}$ & $v_{m}$ & $\Theta_{D}$ \\
\hline glitter & 180.6 & 163.7 & 172.2 & 79.6 & 26.0 & 52.8 & 208.3 & 74.0 & 141.2 & 0.36 & 4573 & 9801 & 5149 & 681 \\
\hline pyrite & 194.0 & 194.0 & 194.0 & 106.1 & 86.9 & 96.5 & 269.2 & 226.8 & 248.0 & 0.29 & 5472 & 10005 & 6102 & 875 \\
\hline $3 \mathrm{C} \mathrm{SiC}$ & & & 225 & & & 192 & & & 448 & 0.168 & 7727 & 12230 & 8502 & 1161 \\
\hline
\end{tabular}

a expt. values for $D F V$ from Daf 12 n.11 nthar volnas darived from these

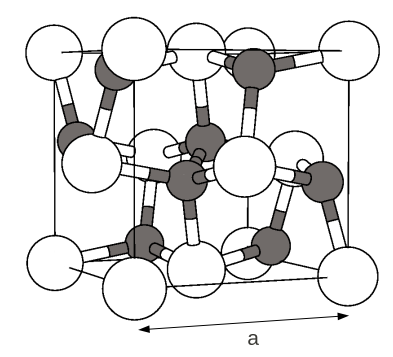

FIG. 1. Pyrite structure with $\mathrm{Si}$ atoms (O) at FCC positions and $\mathrm{C}$ atoms (๑) at $(x, x, x)$ and all symmetry equivalent positions to $(x, x, x)$ where $\mathrm{x}$ is in units of the lattice constant $a$.

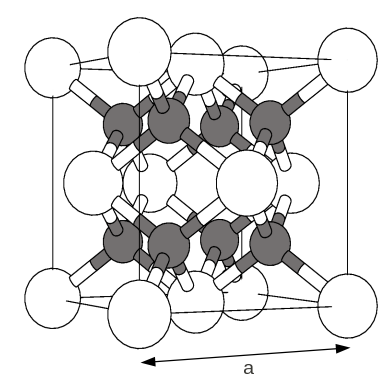

FIG. 2. Fluorite structure: pyrite structure with internal parameter $x=0.25$. Si atoms(O), $\mathrm{C}$ atoms $(\bullet)$. 


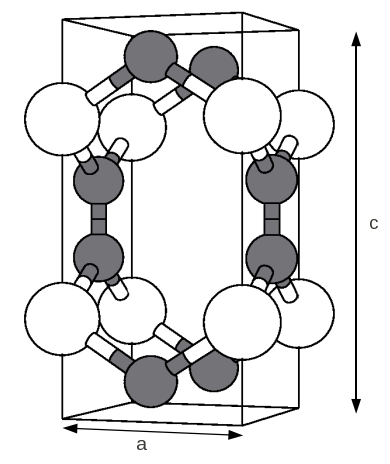

FIG. 3. Glitter structure: Si atoms (O) and C atoms (๑).

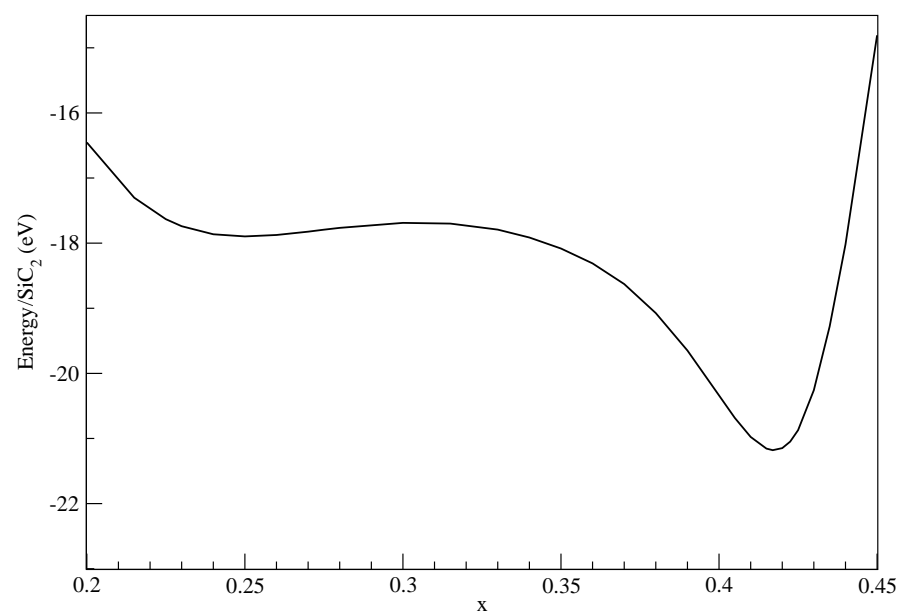

FIG. 4. Total energy as a function of the internal parameter $x$ for the pyrite $\mathrm{SiC}_{2}$ structure showing a global minimum at $x=0.417$ and a local minimum at $x=0.25$.

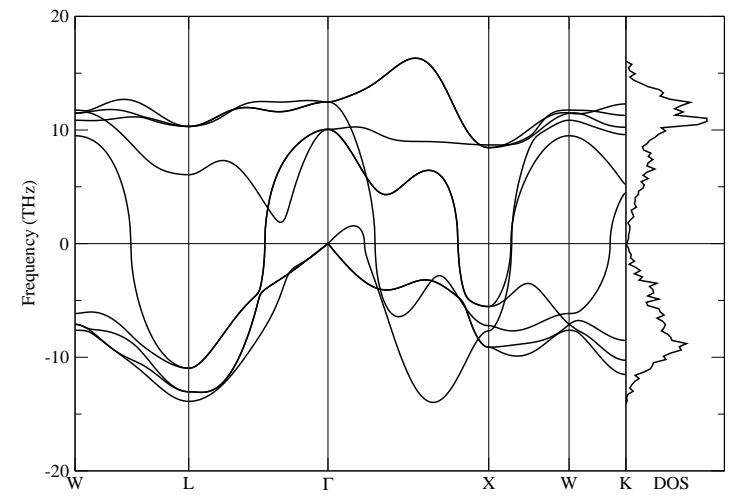

FIG. 5. Phonon dispersion and density of states (DOS) for the fluorite structure showing soft modes. 


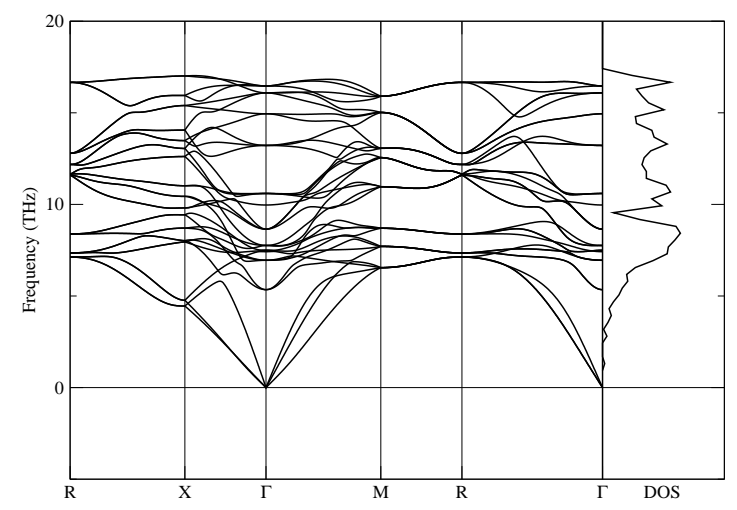

FIG. 6. Phonon dispersion and DOS for the pyrite structure showing no soft modes.

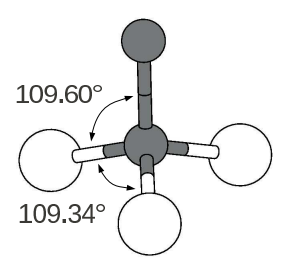

(a)

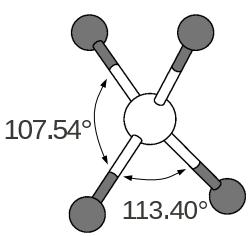

(c)
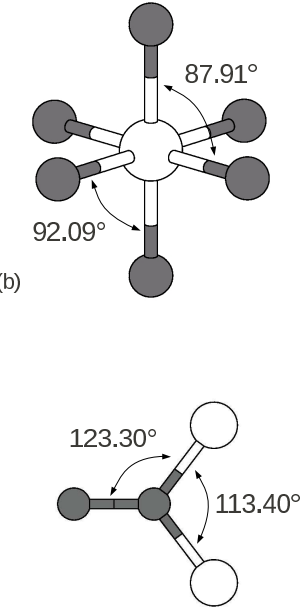

(d)

FIG. 7. Internal angles for the tetrahedral (a) and octahedral (b) bonding in the pyrite structure and the tetrahedral (c) and trigonal (d) bonding in the glitter structure: Si atoms (O) and C atoms (๑).

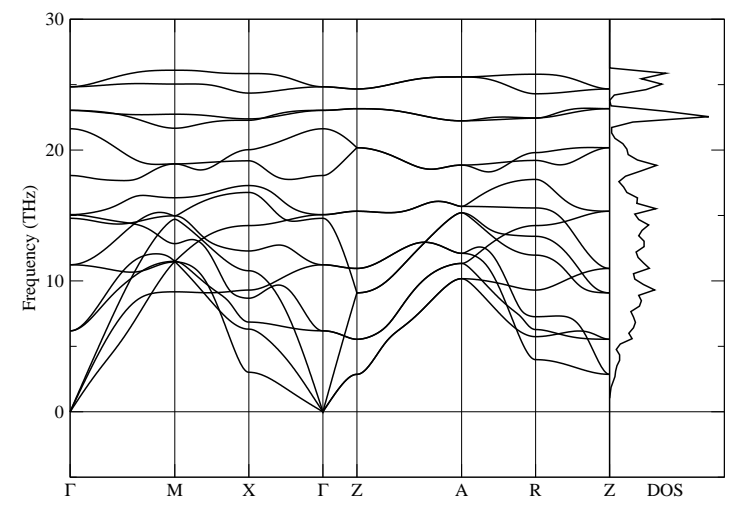

FIG. 8. Phonon dispersion and DOS for the glitter structure showing no soft modes. 


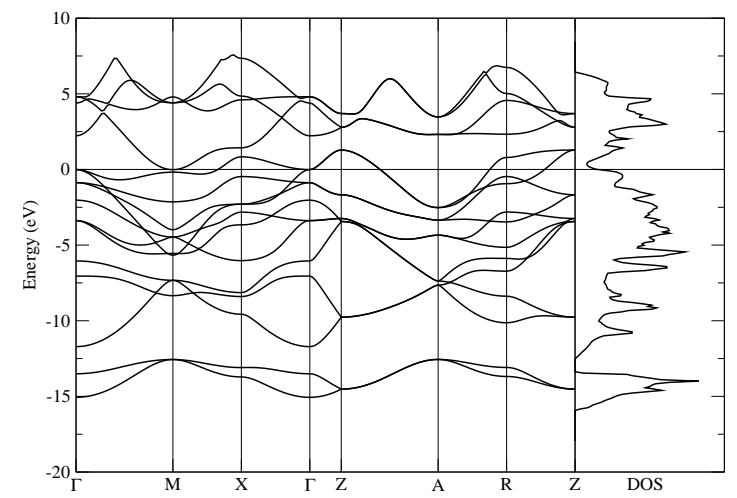

FIG. 9. Band structure and DOS for the glitter structure showing metallic nature and reduced density of states at Fermi level.

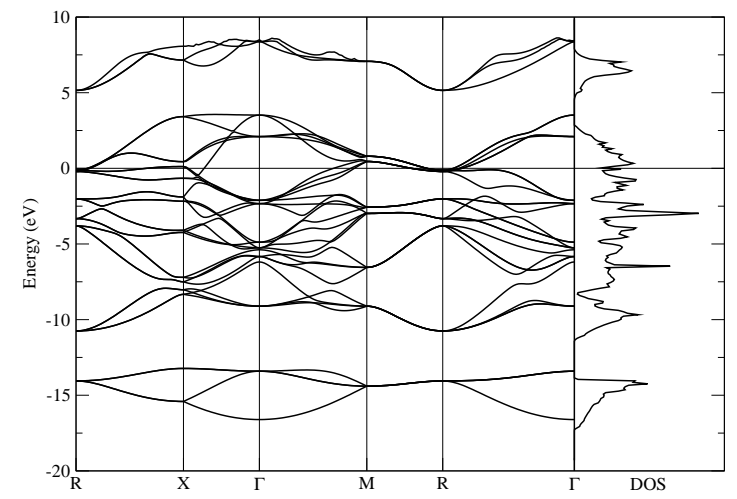

FIG. 10. Band structure and DOS for the pyrite structure showing metallic nature and band-gap above Fermi level.

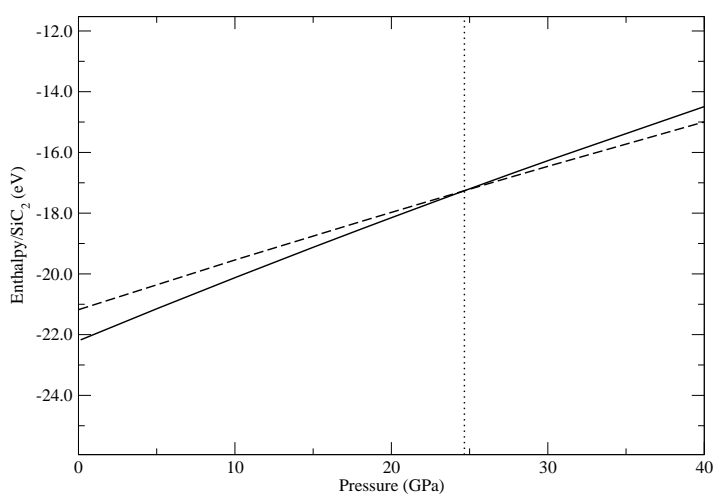

FIG. 11. Enthalpy of the glitter (solid line) and pyrite (dashed line) phases of $\mathrm{SiC}_{2}$ as a function of pressure. The pyrite phase is stable above 24.7 GPa (dotted line). 